independent for each play, even though many of the references are repeated. Thus, a reader of Cornelia is not referred back to Antonius in order to check a mythological reference used in both plays.

Altogether, this volume is an excellent critical edition: solidly researched, sensibly organized, and practical to use. It contains a comprehensive bibliography of the most up-to-date research in both French and English and draws upon this foundation abundantly in its critical commentary. It thereby provides readers with all the background necessary to understand and appreciate the plays, highlights the wide array of questions and studies they have already inspired, and provides an excellent starting point for anyone wishing to explore them in greater depth.

LUKE ARNASON

York University

\title{
Bertoglio, Chiara.
}

\section{Reforming Music. Music in the Religious Reformations of the Sixteenth Century.}

Berlin, Boston : De Gruyter, 2017. xxiii, 836 p. ISBN 978-3-11-051805-4 (relié) 103,99 US\$.

C'est une véritable somme sur la musique des réformes du XVI ${ }^{e}$ siècle que nous offre Chiara Bertoglio, qui s'est donné la tâche de remédier à l'absence d'une étude englobante sur ce thème. L'ouvrage qui compte un peu plus de 800 pages est organisé en douze très denses chapitres, répartis en trois démarches différentes donnant lieu à trois grandes sections implicites.

La première de ces sections, qui compte trois chapitres, consiste en trois études préliminaires nécessaires à ce qui va suivre. Le premier chapitre offre donc un survol du contexte historique. Lauteur y évoque des questions théologiques, culturelles, musicales, socio-politiques et ecclésiastiques, ce qui inclut des courtes descriptions de la vie scientifique, philosophique, littéraire et artistiques de la période. Le deuxième chapitre dans son ensemble examine les différentes façons de voir la musique - dans le contexte du christianisme - du point de vue humaniste et spirituel présentes au XVI ${ }^{e}$ siècle, non pas en prenant les diverses confessions chrétiennes comme repère, mais en distinguant les 
composantes de la tradition menant à ces points de vue. C'est pourquoi ce chapitre semble commencer très lentement : il fait entre autres le lien entre les sources théoriques antiques sur la musique et l'humanisme. Le troisième chapitre consiste en une espèce d'état des lieux en matière musicale sacrée au début du XVI ${ }^{e}$ siècle. L'auteur en profite ici pour discuter de ce qu'est la musique sacrée, afin de mettre en lumière plusieurs autres catégories de musique en lien avec le domaine de la foi, ce qui l'amène à évoquer les diverses expressions de la foi en-dehors de la liturgie, d'abord en général puis en décrivant les formes musicales de piété d'avant Luther. Dans la foulée de cette réflexion, elle traite du statut au même moment historique du plain-chant et de la polyphonie.

La deuxième partie, qui compte cinq chapitres, entre ensuite dans le vif $\mathrm{du}$ sujet, mais encore ici l'auteur procède très graduellement. Le quatrième chapitre présente donc les points de vue de chaque réformateur sur le fait musical. Comme l'auteur l'indique, le point de vue se déplace donc de la musique en général, vers le point de vue spirituel (164), d'un point de vue théorique englobant, vers des points de vue qui conduiront ensuite à des décisions pratiques. L'auteure examine d'abord les idées sur lesquelles les réformateurs et les catholiques s'entendent, en commençant par leur sources philosophiques et théologiques communes, et leur intérêt marqué pour le texte. Une analyse des différents propos réformateurs conduit l'auteure à présenter ensuite les différentes fonctions, vertus et usages qu'ils reconnaissent à la musique sacrée. La deuxième moitié de ce chapitre montre ensuite comment ces prises de positions se retrouvent dans les réflexions entre autres de Luther, puis de Zwingli, de Calvin, et de la réforme anglicane, démarche qui en fait ressortir les différences.

Les trois chapitres suivants examinent ensuite plus précisément les pratiques musicales adoptées par chacune des grandes réformes, en incluant comment s'est constitué les différents répertoires confessionnels. Ainsi, le chapitre 5 présente la réforme luthérienne de la musique en soulignant les sources qu'elle a privilégiée, en décrivant le travail créatif rendu nécessaire, ainsi que le répertoire musical qui en a résulté. Les dernières pages se penchent sur quelques exemples de pratique du répertoire luthérien. Les chapitres 6 et 7 effectuent la même démarche à quelques différences près, pour l'église de Calvin, puis pour l'église d'Angleterre. Le lecteur est ainsi introduit par exemple au choral protestant, aux livres luthériens d'hymnes, aux pratiques de Strasbourg, aux psaumes de Marot, au Psautier de Genève, et au Book of Common Prayer. Les 
derniers paragraphes du chapitre consacré à la musique de l'Église d'Angleterre se penchent sur le cas d'un catholique à la cour anglaise - William Byrd, ce qui sert de belle transition aux deux chapitres suivants examinant ce que l'on a coutume de nommer la Contre-Réforme.

En effet, les chapitres 8 et 9 sont centrés sur le Concile de Trente. Toutefois, d'entrée de jeu, l'auteur précise que ce serait faire fausse route que d'examiner le Concile de Trente et sa postérité uniquement comme une réaction aux mouvements de réformes (382). Le chapitre 8 est organisé en deux parties. La première présente le Concile de Trente dans sa globalité, d'abord en décrivant les préoccupations théologiques en question, puis en présentant les participants. Sur ces derniers, l'auteure donne également leurs positions dans les discussions sur la musique qui sont l'objet de la deuxième partie. La deuxième partie du chapitre se concentre sur les moments du Concile s'étant penchés explicitement sur la musique, ainsi que sur les décisions qui en ont résulté. Du point de vue de ce dernier point, le chapitre 9 est beaucoup plus intéressant, puisqu'il y est question des développements de la musique sacrée catholique, et de ses rapports avec les décisions du Concile. On y constate que la réelle réforme catholique de la musique ne s'est pas faite dans le cadre du Concile, mais à travers plusieurs initiatives ecclésiastiques régionales, individuelles et collectives, telles que la réorganisation jésuite de son éducation et de ses pratiques musicales, et la musique au cœur des pratiques de la Congrégation de l'Oratoire. Il y est question aussi d'efforts de réforme plus généraux tels que la réforme du plainchant, la réduction du répertoire de séquences, et l'établissement graduel d'une « esthétique post-tridentine».

Enfin, la troisième partie de l'ouvrage se penche sur des questions transversales et donc transconfessionnelles, qui n'auraient pu être traitées autrement et dont la transversalité fait leur grand intérêt. Le chapitre 10 explore le rôle de la musique dans la propagande des confessions et dans la construction de l'identité confessionnelle. Après avoir montré comment les confessions se sont opposées par la musique, le chapitre suivant montre comment la musique pouvait aussi être un élément rassembleur traversant ces mêmes confessions, non seulement par ses points communs et par ses objectifs spirituels fondamentaux, mais aussi par la reconnaissance de la valeur musicale et esthétique d'œuvres musicales l'emportant sur les débats théologiques, du moins en ce qui concerne les musiciens, les esthètes, et les humanistes, toutes confessions confondues. Le douzième et dernier chapitre porte sur les femmes 
et la musique dans les contextes confessionnels, ce qui développe en plus grand détails certaines décisions du Concile de Trent évoquées dans le chapitre 8. Toutefois, ce lien organique avec le thème général masque le fait que ce chapitre correspond en réalité à une étude bien développée sur les femmes et la musique à la Renaissance qui peut servir de point de départ à qui voudrait étudier davantage la question. En effet, on y fait par exemple le tour de toutes les grandes figures féminines - anonymes ou non - de la musique sacrée du $\mathrm{XVI}^{\mathrm{e}}$ siècle.

Cet ensemble est encadré d'une part par deux préfaces (de Jeremy S. Begbie puis de Sir James MacMillan), une table des matières très subdivisées permettant une consultation rapide, et d'autre part, par un glossaire, une bibliographie encyclopédique divisée en sources primaires et secondaires et par un index des noms et un index des sujets. Seule réserve, le fait que la bibliographie de sources secondaires comptant 54 pages de titres de langues anglaise, italienne et allemande n'incluent qu'une vingtaine de titres en français laisse assez perplexe.

Le travail de Chiara Bertoglio se démarque par plusieurs idées, prises de positions, et caractéristiques qu'elle fait délibérément, il me semble, ressortir. Dès l'introduction, elle souligne le fait que la période et les idées étudiées dans son ouvrage sont complètement étrangères à notre vie post-moderne (xxvi). Elle souligne par là le caractère intrinsèquement idéaliste de l'entreprise historienne. Elle répond à cette difficulté en réitérant régulièrement la nécessité de faire des nuances et de ne pas privilégier un point de vue au dépend des autres ; par exemple, en ne préférant pas une confession plutôt qu'une autre, en n'émettant pas de jugement de valeur sur des musiques savantes au dépend de musiques qui le sont moins, et en excluant le moins possible une approche disciplinaire au profit d'une autre (51). L'ouvrage qui en résulte n'a donc pas un caractère encyclopédique seulement par son contenu et les thèmes abordés, mais aussi par les savoirs disciplinaires mis à profit. Il devient également un exemple d'une grande discipline intellectuelle à l'œuvre qui vise toujours à se frayer un chemin entre les extrêmes des spectres d'idées présents à notre esprit. L'auteur émet également l'idée que l'étude de la musique devrait nous entraîner à s'écouter les uns les autres et donc à plus de respect et d'urbanité (xxviii), ce qui peut être directement mis en lien avec ce qui précède. Elle présente également son entreprise comme ayant un objectif de réconciliation des confessions chrétiennes par la musique. Ces idées exprimées essentiellement dans son introduction générale donne un ton politique à l'exercice de la musicologie qui 
ne déplaît pas. Au niveau du contenu, ce que j’ai par ailleurs le plus apprécié au fil des pages s'avère être une discussion en filigrane qui graduellement se dégage de tout le livre et qui pose la question du rôle de la polyphonie et qui donne lieu à quelques paragraphes bien sentis sur l'expérience spirituelle, voire mystique, que favorise la polyphonie

L’ouvrage est surtout conçu pour donner un point de départ à de nouvelles recherches. Toutefois, plusieurs de ses aspects rend l'ouvrage particulièrement utile aux étudiants et aux enseignants. Tout d'abord l'ouvrage complet est si bien organisé qu'il est très facile à consulter. L'auteur explicite systématiquement en début et en fin de chacune de ces sections ses objectifs et les étapes de sa démarche. Enfin, les expositions et la langue, sans compromis pour les contenus plus complexes, sont toujours très claires et efficaces. C'est pourquoi cet ouvrage de Chiara Bertoglio n'est pas seulement nécessaire et remarquable, il est également une leçon de musicologie.

PASCALE DUHAMEL

Université d'Ottawa

Blanchard, W. Scott, and Andrea Severi, eds.

Renaissance Encyclopaedism: Studies in Curiosity and Ambition.

Essays and Studies 41. Toronto: Centre for Reformation and Renaissance Studies, 2018. Pp. 467. ISBN 978-0-7727-2189-1 (paperback) CDN\$49.95.

Understanding a "Renaissance man" as a polymath possessed of universal knowledge is a modern commonplace, but we do not fully appreciate how Renaissance humanists regarded encyclopaedic achievements. Scott Blanchard and Andrea Severi have brought together many contributors in Renaissance Encyclopaedism to examine how Renaissance ideas of scholarship, harmony, and utility drove the collection and categorization of information. This volume comes at an important time in the advancement of cultural studies in general as well as encyclopaedism in particular. Each chapter focuses on individual Renaissance figures or contexts coming out of a long Quattrocento running from the time of Coluccio Salutati through the age of Desiderius Erasmus and Guillaume Budé. The eleven essays embody the qualities of curiosity and 